\title{
A microfluidic bleeding model to investigate the effects of blood flow shear on microvascular hemostasis
}

\author{
Xiangyu HU ${ }^{1}$, Haosheng CHEN ${ }^{1}$, Jiang $\mathrm{LI}^{2}$, Kuilin MENG ${ }^{1}$, Yuming WANG ${ }^{1}$, Yongjian $\mathrm{LI}^{1, *}$ \\ ${ }^{1}$ State Key Laboratory of Tribology, Tsinghua University, Beijing 100084, China \\ ${ }^{2}$ School of Mechanical Engineering, University of Science and Technology Beijing, Beijing 100083, China \\ Received: 22 July 2020 / Revised: 22 October 2020 / Accepted: 03 November 2020 \\ (C) The author(s) 2020 .
}

\begin{abstract}
Hemorrhage is the phenomenon of blood loss caused by vascular trauma or other pathological reasons, which is life-threatening in severe cases. Because microhemorrhage is difficult to visually monitor and pre-treat in vivo, it is necessary to establish in vitro prediction methods to study the hemostasis mechanism in different physiological environments. In this study, a microfluidic bleeding model was developed to investigate the effect of blood flow shear on microvascular hemostasis. The results indicated that the regulation of blood shear rate on platelet aggregation affected the growth and morphology of hemostatic thrombus, and finally regulated the process of hemostasis. This in vitro model is significant to studies on hemostatic mechanisms, a reliable prediction of microhemorrhages, and an adjustment of the treatment scheme.
\end{abstract}

Keywords: microfluidic; hemostasis; platelet accumulation

\section{Introduction}

Hemorrhage is the phenomenon of blood loss caused by vascular trauma or other pathological reasons, which can endanger life in severe cases [1,2]. Compared to a ruptured macrovascular hemorrhage, a microhemorrhage, such as a gastrointestinal [3], hepatorrhagia [4], and cerebral hemorrhage [5], is also often fatal. Anticoagulant treatment, hypertension, advanced age, and violent mood fluctuations, among other factors, can easily cause a microhemorrhage, particularly cerebral hemorrhage [6,7]. Microhemorrhage in the body can be subtle but dangerous. Some gastrointestinal microbleeds are self-healing, but there is also a risk of acute massive bleeding that requires endoscopic therapy such as hemostatic forceps, high-frequency radio coagulation, and thrombin injections [3]. Cerebral hemorrhage is difficult to control with external hemostatic intervention, and can only be regulated by indirect means, such as reducing the intracranial pressure [7-9]. This is more dependent on the physiological coagulation mechanism required for hemostasis.

Hemostasis is a physiological response to vascular injury, during which blood clots form to seal the wound and prevent blood loss [1]. The formation of a hemostatic plug at sites of vascular injury relies on the dynamical processes of platelet accumulation and activation of the coagulation cascade in the setting of the biorheology of blood flow [10]. When the vessel is broken, the exposure of the blood to the subendothelial matrix initiates platelets adhering to the wound site through the von Willebrand factor (vWF) and collagen or other extracellular matrix protein binding, under hemodynamic shear conditions of the blood flow [11, 12]. The adhesion activates the platelets, which then triggers a suite of responses, including the mobilization of binding receptors on the platelet's surface and the release of chemical agonists into the blood plasma [13]. These agonists

* Corresponding author: Yongjian LI, E-mail: liyongjian@tsinghua.edu.cn 
induce more active platelets, and the activated platelets bind together to form a platelet embolism, which is called platelet accumulation. The activation of platelets and the exposure of the tissue factor (TF) also initiate a coagulation cascade [1, 2]. Thrombin is the major product of coagulation, which converts the soluble plasma protein fibrinogen into insoluble polymerized fibrin to form a stabilizing fibrin mesh surrounding the deposited platelets. Finally, a stable hemostatic plug is formed [2].

Because microhemorrhages are difficult to visually monitor and pre-treat in vivo, it is necessary to establish in vitro prediction methods to study hemostasis mechanisms in different physiological environments, which is significant for reliably predicting a microhemorrhage and adjusting the treatment scheme [14, 15]. In medicine, experimental diagnostic methods for hemorrhagic diseases mainly include the lengths of the bleeding, coagulation, the activation of partial thromboplastin, prothrombin, and thrombin [16]. These diagnostic methods can predict hemorrhagic disease to a certain extent, but in vitro single-factor monitoring ignores the physiologic flow shear environment of hemostasis problems. Some in vivo animal hemostasis models show the direct global process of hemostasis at the wound sites, but it is difficult to apply mechanistic research at the intercellular level, which is limited by the means of observation and variable control $[17,18]$. Owing to the recent advances in microfabrication technologies, microfluidic platforms have been extensively used for microscale biological and biochemical experiments, owing to their low cost, high throughput, and good biocompatibility $[15,19]$. In vitro microfluidic models have been widely used to study the mechanism of coagulation $[2,20]$ or intravascular thrombosis [21, 22]. However, the mechanism of intravascular thrombosis may not directly translate into extravascular thrombosis, which is relevant to bleeding blockage and hemostasis $[23,24]$. In vitro hemostasis models have been studied relatively little. Schoeman et al. [23] presented a microfluidic hemostasis model to assess the bleeding time and explore the platelet function. To investigate the interaction of different components of hemostasis under altered microenvironments, Sakurai et al. [24] devised an ingenious microfluidic system coupled with a microengineered pneumatic valve that induces a vascular "injury". However, both studies only focused on major biological or biochemical mechanisms of hemostasis and ignored the role of blood flow in regulating the process of hemostasis. A number of studies have shown that local flow conditions have a crucial effect on the coagulation and thrombosis processes [25-27]. Therefore, it is necessary to construct a microvascular hemostasis model in vitro to study the regulation of blood flow in hemostasis.

Given this background, a microfluidic bleeding model was developed to investigate the effect of blood flow shear on microvascular hemostasis in this study. The injury channel was coated with Type I collagen to mimic the subendothelial matrix exposed during vascular trauma and promote platelet adhesion and coagulation. The blood flow is controlled to mimic the flow shear bio-environment from venous to arteriole (average wall shear rate of $100-2,000 \mathrm{~s}^{-1}$ ). In this model, the entire process of microvascular hemostasis can be monitored in real time, the hemostasis time is predicted well, and the regulation mechanism of blood flow shear on hemostasis is revealed. In conclusion, this microvascular hemostasis model provides an ideal platform for studying human blood samples and predicting hemostatic and thrombotic diseases in vitro.

\section{Materials and methods}

\subsection{Reagents and antibodies}

Human CD41 monoclonal antibody FITC conjugate and fibrinogen from human plasma Alexa Fluor 594 conjugate were purchased from Thermo Fisher Scientific (China) Inc., Shanghai, China. Type I collagen was purchased from Yeasen Biotechnology Co., Shanghai, China. A sodium citrate buffer, DiOC, and Dil were purchased from Solarbio Co., Beijing, China. Anti-human vWF antibody conjugated with fluorescein isothiocyanate (FITC) was purchased from Rockland Immunochemicals Inc., Limerick, USA. N-Acetyl-L-cysteine and bovine serum albumin (BSA) were purchased from Sigma-Aldrich (Shanghai) Co., 
Shanghai, China. Eptifibatide was purchased from Shanghai Macklin Biochemical Co., Shanghai, China.

\subsection{Microfluidic device design and construction}

The microfluidic devices used in this study were fabricated with polydimethylsiloxane (PDMS, Dow Corning) using soft lithography techniques [28]. In brief, the microfluidic channel mold made of SU-8 (Microchem, Co.) was patterned on a silicon wafer using ultraviolet lithography (SF100, Advanced Micro Patterning, Co.). The PDMS prepolymer was mixed with the curing agent at a 10:1 ratio (w/w) and poured onto the patterned silicon wafer template. The mixture was cured in an oven at $70{ }^{\circ} \mathrm{C}$ for $1.5 \mathrm{~h}$ and peeled carefully from the wafer. Inlet holes (diameter $=1 \mathrm{~mm}$ ) were defined using a biopsy puncher on the PDMS layer. The interior surfaces of the PDMS layer, which had been oxidized by exposure to plasma, were bonded to glass slides cleaned in 1:1 $12 \mathrm{M} \mathrm{HCl}$ :methanol for $1 \mathrm{~h}$, rinsed with 18.2 M $\Omega-\mathrm{cm}$ deionized water and subsequently dried using high-pressure air.

The microfluidic channel was designed in the shape of the letter ' $\mathrm{H}$ ' where the outer two horizontal channels represent the vascular (blood perfusion channel) and extravascular compartments (buffer flush channel), respectively, and the horizontal channels are connected using a vertical channel representing the vascular wound (Fig. 1(b)) [23]. The width of the blood perfusion channel and the buffer flush channel was $200 \mu \mathrm{m}$. The width of the vascular wound channel was $50 \mu \mathrm{m}$, and the length was $150 \mu \mathrm{m}$. The channel section has a consistent height of $50 \mu \mathrm{m}$. The microfluidic bleeding model a

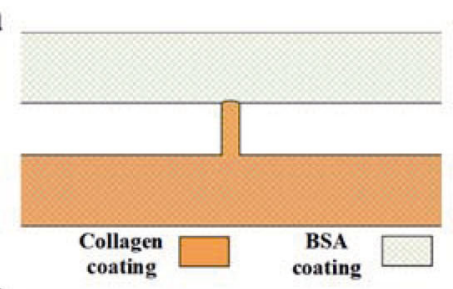

d
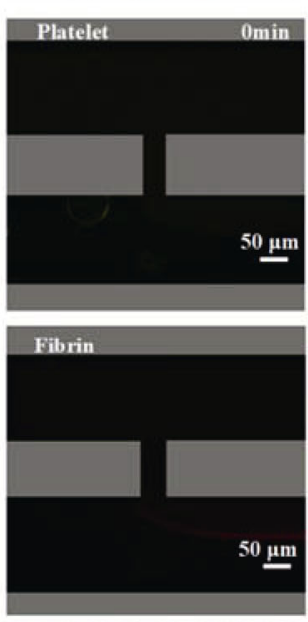

$50 \underline{\mu m}$

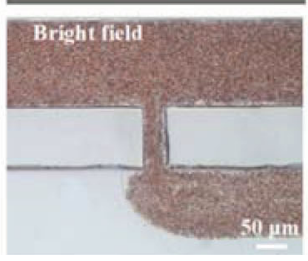

b
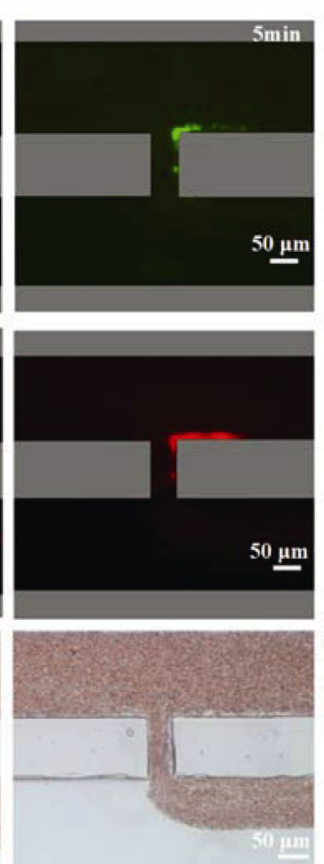

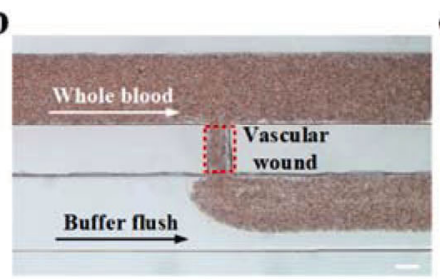

c


Fig. 1 Microfluidic bleeding model of microvascular hemostasis: (a) Schematic of collagen coating layer preparation and main channel anticoagulation; (b) bright field diagram of whole blood perfusion experiment; (c) blood flow pattern detected using $\mu$ PIV. (d) Real-time visualization of the entire hemostatic process from the $1,500 \mathrm{~s}^{-1}$ group.. Fluorescent label: platelets (green) and fibrin (ogen) (red). 
in this study applies to the microhemorrhage phenomenon occurring in the microcirculation system, such as gastrointestinal, hepatorrhagia, and cerebral hemorrhages because the microvessels that are distributed at these microhemorrhage sites have diameters ranging from tens to hundreds of microns [3-6, 23-24]. The microscale of the microfluid bleeding model can present a stable laminar flow, which enables the shear stress to be strictly controlled and regulated, thus achieving the dynamic physiological range of the microcirculation.

Type I collagen $\left(0.5 \mathrm{mg} \cdot \mathrm{ml}^{-1}\right)$ [22] was adsorbed into the walls of the vascular wound channel, as shown in Fig. 1(a). Using a pipette, a $10-\mu \mathrm{L}$ collagen solution was drained and slowly injected into the inlet of the buffer flush channel while the blood perfusion channel was empty. The wound channel was then filled, although the solution was contained in the wound channel owing to capillary forces at the solution-air interface between the wound and blood channels. Collagen solutions were allowed to adsorb on the walls of the wound channel at $4{ }^{\circ} \mathrm{C}$ for $12 \mathrm{~h}$. Prior to the perfusion of blood, the blood channel was blocked with $2 \%$ BSA in phosphate buffer solution (PBS) for $1 \mathrm{~h}$ to increase the anticoagulation.

\subsection{Blood collection and preparation}

Whole blood was collected from healthy donors using a venepuncture and stored in vacutainer tubes containing 3.2\% sodium citrate to prevent coagulation. All procedures involving human participants were conducted in compliance with the relevant laws and guidelines approved by the Institutional Review Board (IRB) of Tsinghua University (No. 20200036), and with the 1964 Helsinki declaration and its later amendments. Alexa-594 labeled human fibrinogen (final concentration of $12.5 \mu \mathrm{g} \cdot \mathrm{mL}^{-1}$ ) was added to the collected blood to visualize the deposition of the fibrin. Platelets were labeled with an FITC-conjugated CD41 monoclonal antibody (final concentration of $5 \mu \mathrm{g} \cdot \mathrm{mL}^{-1}$, with green fluorescence) or Dil (final concentration of $1 \mu \mathrm{g} \cdot \mathrm{mL}^{-1}$, with red fluorescence). vWF was stained with FITCconjugated vWF antibody (final concentration of $\left.1 \mu \mathrm{g} \cdot \mathrm{mL}^{-1}\right)$. The labeled blood was incubated at $37{ }^{\circ} \mathrm{C}$ in the presence or absence of eptifibatide $\left(50 \mu \mathrm{g} \cdot \mathrm{mL}^{-1}\right)$ or NAC (30 mM) for $15 \mathrm{~min}$ prior to the assay. Before the perfusion experiment, the labeled blood was treated by rapidly mixing the blood with a recalcification buffer at a ratio of 9:1 to restore the coagulation, which was prepared by dissolving $75 \mathrm{mM}$ of $\mathrm{CaCl}_{2}$ and $35 \mathrm{mM}$ of $\mathrm{MgCl}_{2}$ in a PBS. During the experiment, labeled recalculated whole blood was perfused into the blood perfusion channel at a controlled flow rate $\left(0.5,2.5,5,7.5,10 \mu \mathrm{L} \cdot \mathrm{min}^{-1}\right)$ to simulate the average wall shear rate $(100,500,1,000,1,500$, and 2,000 s $\mathrm{s}^{-1}$ ) of blood flow under different physiological conditions from the veins to the arteries. A sodium citrate buffer was perfused into the buffer flush channel at half the blood flow rate to simulate the extravascular compartments.

\subsection{Measurement system and methods}

Red blood cells (RBCs) with a hematocrit of $0.5 \%$ in PBS were directly used as tracer particles to show the flow field in a bright field under an inverted microscope (IX83, Olympus Co.). The motion of the RBCs was recorded using a high-speed camera (M110, Phantom Co.). The recorded videos were analyzed using $\mu$ PIV software (DynamicStudio, Dantec Co.) to acquire the velocity field in the microfluidic bleeding model (Fig. 1(c)). The hemostasis processes under different flow conditions were measured using a fluorescence microscope (IX83, Olympus Co.). Image J (National Institutes of Health) was used to calculate the coverage area of hemostatic thrombosis in the wound channel.

\section{Results and discussion}

\subsection{Characterization of in vitro microfluidic bleeding and hemostasis model}

A polydimethylsiloxane (PDMS)-based microfluidic device was used to simulate in vitro microvascular bleeding. Two horizontal channels of the " $\mathrm{H}$ " shaped microfluidic channel represent the vascular (blood perfusion channel) and extravascular compartments (buffer flush channel), respectively, and the vertical connection channel represents the vascular wound (Fig. 1(b)). Physiologically, damaged blood vessel walls expose the subendothelial matrix rich in 
adhesive and procoagulant proteins that support thrombus formation. In this model, the internal surface of the wound channel is precoated with Type I collagen, which is abundant in the subcutaneous matrix, and the blood channel was precoated with BSA (Fig. 1(a)). Type I collagen is a strong agonist and adhesive ligand for platelets through the GP and integrin $\alpha_{2} \beta_{1}$ [29]. The endothelial surface of the blood vessel is replaced by a BSA layer coated on the internal surface of the blood channel, which played a certain antithrombotic role [23]. During the experiment, re-calcified sodium citrate-treated whole blood was perfused through the device at a controlled flow rate, and the average wall shear rate achieved $\left(100,500,1,000,1,500,2,000 \mathrm{~s}^{-1}\right)$ can approximately simulate the blood shear environment within the physiological range from the veins to the arteries and arteriloes $[2,12]$. According to the $\mu$ PIV flow field analysis (Fig. 1(c)), the blood flow forms a high-speed flow area in the inlet and outlet of the wound channel, which will wash away the hemostatic thrombus formed and reduce the local concentration of procoagulant substances. Real-time monitoring of hemostasis can then be performed through fluorescence microscopy, and the hemostasis time (defined as the time at which red blood cells stop transiting into the extravascular area) and fluorescence coverage area of relevant biological species are then monitored. Figure 1(d) shows the whole process of hemostatic thrombus from initiation and growth up to hemostasis achieved under a flow condition of $1,500 \mathrm{~s}^{-1}$. Because the blood is continuously perfused into the blood channel, it also flows through the wound channel into the extravascular channel, similar to "bleeding" (Fig. 1(b)). As blood flows through the wound channel, platelets adhere throughout the mimic injury site, and platelet aggregation and fibrin polymerization lead to the formation of a hemostatic plug at the wound site. The hemostatic thrombus continues to grow until the wound channel is blocked and the bleeding stops (Fig. 1(d)). In this group, the hemostasis time was $15.5 \mathrm{~min}$.

\subsection{Process of hemostasis under different blood flow shear rates}

The hemostasis time and hemostatic process under different shear rates are shown in Fig. 2. In the hemostasis model of the same size, when the shear rate of blood flow increased from 100 to 2,000 s-1, the hemostasis time changed, as shown in Fig. 2(a). It can clearly be seen that, at the lowest $\left(100 \mathrm{~s}^{-1}\right)$ and highest $\left(2,000 \mathrm{~s}^{-1}\right)$ shear rates, the hemostasis time was relatively short, at approximately 17.0 and 14.2 min, respectively. However, at the intermediate shear rate $\left(1,000 \mathrm{~s}^{-1}\right)$, the wound cannot be completely blocked after $30 \mathrm{~min}$, and hemostatic failure occurs. Figures 2(b) and 2(c) show the fluorescence coverage of platelets and fibrin in hemostatic thrombus at different shear rates with an increase in the hemostatic time. Figure 2(d) shows the area fraction of hemostatic thrombus at different shear rates when hemostasis was achieved (data at $1,000 \mathrm{~s}^{-1}$ was obtained after blood perfusion for $30 \mathrm{~min}$ ). As can be seen, when the shear rate increased from 100 to $1,000 \mathrm{~s}^{-1}$, the coverage area of the platelets and fibrin in the hemostatic thrombus gradually decreased; when the shear rate increased from 1,000 to $2,000 \mathrm{~s}^{-1}$, the coverage area of both increased gradually. The growth rate of hemostatic thrombus was also affected by shear rate. At an intermediate shear rate $\left(1,000 \mathrm{~s}^{-1}\right)$, the coverage area of hemostatic thrombosis was the smallest, the growth rate was the slowest, the hemostatic time was the longest, and a hemostatic failure occurred. At a low shear rate $\left(100 \mathrm{~s}^{-1}\right)$ similar to a venous environment, the fibrin coverage is the largest, the growth is the fastest, and the hemostasis time is shorter. At a high shear rate $\left(2,000 \mathrm{~s}^{-1}\right)$ similar to the arteriole environment, the platelet coverage area is the largest, the growth rate is the fastest, and the hemostasis time is the shortest.

Because the geometric size of the hemostasis model is unchanged, with an increase in the shear rate, the scouring effect of blood flow on the wound channel and the dilution effect on the local concentration of coagulant should be gradually enhanced. However, the occurrence of an intermediate shear rate, which is prone to hemostatic failure, indicates that the hemostatic mechanism is also influenced and regulated by the shear rate of blood flow. It is clearly shown in Fig. 2(d) that the coverage of fibrin in each experimental group was not significantly different. Except for the low coverage fraction (approximately $60 \%$ ) in the hemostasis failure group, the coverage 

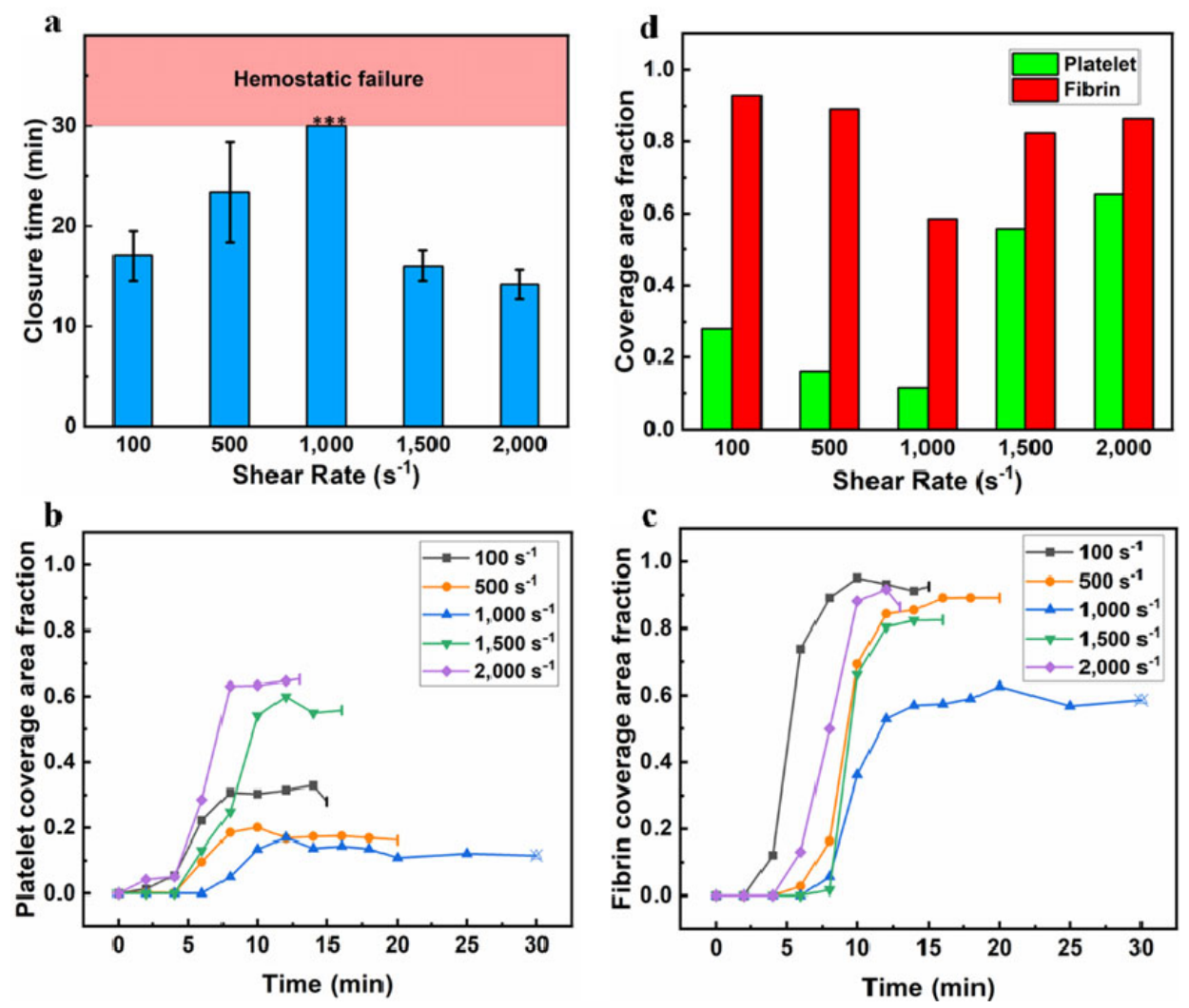

Fig. 2 (a) Wound closure time at different shear rates of blood flow; (b) growth of hemostatic thrombus at different shear rates (platelet and fibrin coverage area fraction; "|" indicates that hemostasis is achieved, and " $\times$ " and hollow symbols indicate a hemostasis failure); (c) growth of hemostatic thrombus at different shear rates (fibrin coverage area fraction); (d) area fraction of hemostatic thrombus at different shear rates when hemostasis is achieved. It should be noted that the data at $1,000 \mathrm{~s}^{-1}$ was obtained after blood perfusion for $30 \mathrm{~min}$, indicating a hemostatic failure.

fractions in all other groups were more than $80 \%$. However, there were significant differences in platelet coverage between groups. These results suggest that the difference in the hemostasis process under different flow shear rates is related to the platelet aggregation mechanism. The propensity of platelets to clump together at sites of vascular injury is described as platelet aggregation, and it is now widely accepted that one of the key elements influencing the platelet aggregation process is blood flow, with evidence that distinct aggregation mechanisms operate under different shear conditions [12, 30]. The following sections describe how the shear-condition-mediated platelet aggregation affects the hemostasis process.

\subsection{Mechanism of flow-mediated platelet accu- mulation on microvascular hemostasis}

Based on findings from in vitro perfusion experiments $[10,22,24]$ and in vivo thrombosis models $[18,26]$, multiple adhesion receptors and ligands are involved in platelet aggregation, the most important of which include vWF, fibrinogen, and integrin $\alpha_{\mathrm{IIb}} \beta_{3}$. Here, $\mathrm{vWF}$ is a large multimeric protein essential for platelet adhesion under arterial flow conditions. The potential shear-dependent changes in the globular conformation of vWF can lead vWF to unfold upon the application of the fluid shear and increase the availability of receptor binding sites for platelets [13, 25, 26]. Integrin $\alpha_{\mathrm{IIb}} \beta_{3}$ is a membrane-bound platelet receptor, and fibrinogen acts as a soluble adhesive protein; the interactions between them have also been demonstrated to support platelet aggregation [26, 30]. The relative effects of each mechanism on the hemostasis process under major shear conditions are experimentally studied.

The distribution of platelets and VWF in hemostasis thrombus after $15 \mathrm{~min}$ of blood perfusion at 100, 1,000 , and $2,000 \mathrm{~s}^{-1}$ was experimentally observed to 
investigate the role of $\mathrm{vWF}$ in platelet aggregation under different shear conditions. It is clearly demonstrated in Fig. 3(a) that, as the shear rate of blood flow increases, vWF gradually dominates the platelet aggregation. At $100 \mathrm{~s}^{-1}$, platelets aggregated in the middle of the wound channel and eventually formed a blocking embolism, but no vWF distribution was found, suggesting that vWF did not play a role in platelet aggregation at a lower shear rate. At 1,000 s-1, platelets accumulated in the medial wall and downstream of the wound channel, but did not form a complete clumpy embolism to block the channel. The vWF distribution area is slightly narrower than that of the platelets, suggesting that vWF is partially involved in platelet aggregation at the intermediate shear rate. In fact, the distribution of platelets and vWF in this group remained in this state until the perfusion experiment was carried out for $30 \mathrm{~min}$, and hemostasis was not achieved. At 2,000 s-1, a large number of platelets accumulated in the interior and outlet of the wound channel, eventually completely blocking the wound channel. vWF is also present in large numbers, and the distribution area basically overlaps with the platelets, which means that vWF dominates the platelet aggregation at a higher shear rate.

From the results shown in Fig. 3(a), it can be inferred that vWF dominates the platelet aggregation in a high-shear state, whereas in a low-shear state, it is most likely dominated by another mechanism, integrin $\alpha_{\mathrm{IIb}} \beta_{3}$-fibrinogen interactions. Large number of previously published studies on thrombosis have

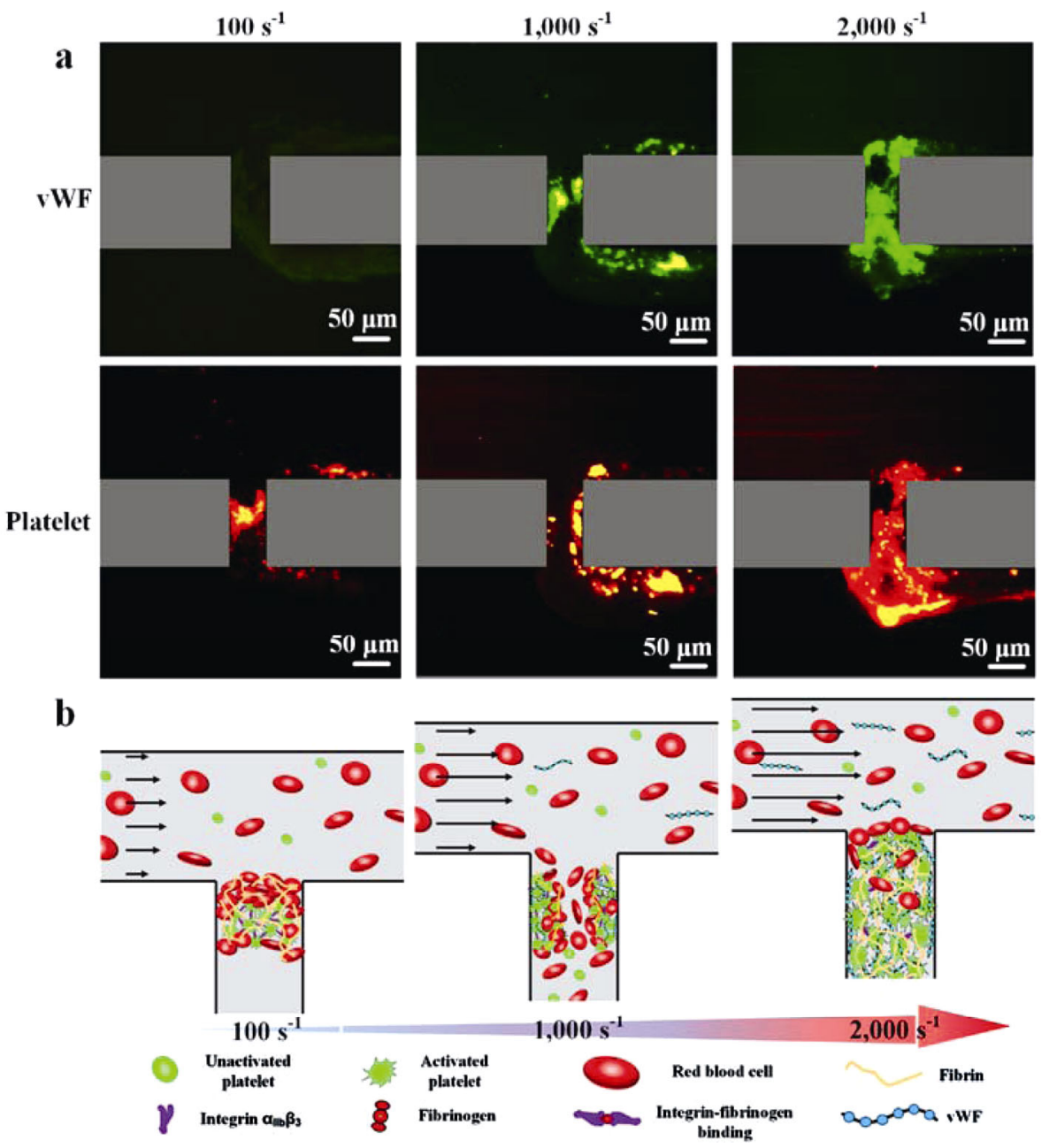

Fig. 3 (a) As the shear rate of blood flow increases, vWF gradually dominates the platelet aggregation; fluorescent label: vWF (green) and platelet (orange red), respectively. (b) Mechanism of flow-mediated platelet accumulation on microvascular hemostasis. 
shown that the platelet aggregation mechanism is regulated by the blood flow shear [25-27, 31]. Studies on the mechanism of platelet aggregation $[26,30]$ indicate that, under conditions of relatively low shear $\left(0-1,000 \mathrm{~s}^{-1}\right)$, platelet aggregation is primarily mediated by soluble fibrinogen, which physically cross-links platelets through the engagement of integrin $\alpha_{\mathrm{IIb}} \beta_{3}$, which is affected by the local concentration of fibrinogen; in addition, at progressively higher wall shear rates $\left(>1,000 \mathrm{~s}^{-1}\right)$, the aggregation becomes more vWF-dependent, and plasma vWF binds to the platelet receptor $\mathrm{GP}_{\mathrm{Ib}}$, leading to platelet activation and subsequent shear-induced platelet aggregation. Studies of plasma fibrinogen and platelet integrin $\alpha_{\mathrm{IIb}} \beta_{3}[32,33]$ support the above viewpoints, suggesting that under relatively low shear conditions $\left(<1,000 \mathrm{~s}^{-1}\right)$, integrin $\alpha_{\mathrm{IIb}} \beta_{3}$ on the surface of free-flowing platelets can engage fibrinogen adsorbed onto the surface of the thrombus; in addition, the subsequent stimulation of platelets by locally generated soluble agonists induces a platelet shape change and an increase in integrin $\alpha_{\mathrm{IIb}} \beta_{3}$ affinity, which helps stabilize and sustain integrin $\alpha_{\mathrm{IIb}} \beta_{3}$-fibrinogen bonds. Studies on plasma vWF $[25,27,31]$ also showed that, at a low shear of $<30 \mathrm{dyn} \cdot \mathrm{cm}^{-2}$ (converted into a shear rate of $\sim 187 \mathrm{~s}^{-1}$, when considering that the blood viscosity in this model is $1.6 \times 10^{-2} \mathrm{~Pa} \cdot \mathrm{s}$, which is estimated from the empirical relationship for blood flow in tubes with diameters of $<1 \mathrm{~mm}$ [34]), vWF remains inactive. When the shear increases beyond $80 \mathrm{dyn} \cdot \mathrm{cm}^{-2}\left(\sim 500 \mathrm{~s}^{-1}\right)$, vWF polymerization occurs and begins to induce platelet aggregation; the mechanisms mediated by integrin $\alpha_{\mathrm{IIb}} \beta_{3}$ and VWF are not mutually exclusive, and both operate within the intermediate shear range; at an extremely high pathological shear level of $>$ $300 \mathrm{dyn} \cdot \mathrm{cm}^{-2}\left(\sim 1875 \mathrm{~s}^{-1}\right)$, the relative contribution of vWF to thrombosis increased significantly and eventually replaced integrin $\alpha_{\mathrm{IIb}} \beta_{3}$ as the main mechanism.

From the above information, it can be seen that the regulation of blood shear rate on platelet aggregation affects the growth and morphology of hemostatic thrombus, and finally regulates the process of hemostasis. The detailed mechanism is shown in Fig. 3(b). At a low shear rate $\left(100 \mathrm{~s}^{-1}\right)$ similar with the venous environment, the dominant mechanism is an integrin $\alpha_{\mathrm{II}} \beta_{3}$-fibrinogen interaction. The integrin $\alpha_{\mathrm{II}} \beta_{3}$-fibrinogen bond is a long-lived, powerful bond with a small $k_{\text {off }}$ (dissociation rate) of $0.15-0.25 \mathrm{~s}^{-1}$ under the action of dissociation produced by a low shear flow $[12,35]$. Under this condition, platelets move slowly and have sufficient response time to form this powerful bond. Therefore, an integrin $\alpha_{\mathrm{IIb}} \beta_{3}$-fibrinogen bond can mediate the formation of a strong adhesion of platelets on the surface of the wounds. The slow blood flow is also conducive to the local increase in the concentration of fibrinogen and other coagulants in the wound channel, and the platelets can aggregate in clumps to form stable hemostatic thrombi. At an intermediate shear rate $\left(1,000 \mathrm{~s}^{-1}\right)$, platelets move faster and do not have an adequate response time, and fibrinogen and other coagulants have difficulty maintaining a relatively high concentration in the wound channel at an increasing flow rate, and thus the integrin $\alpha_{\mathrm{IIb}} \beta_{3}$-fibrinogen mechanism has difficulty in playing a good role. At the same time, this shear rate also makes it difficult for the vWF-mediated mechanism to fully play its role, and thus it is difficult for platelets to aggregate to block the entire channel, and a hemostasis failure is likely to occur. At a high shear rate $\left(2,000 \mathrm{~s}^{-1}\right)$ similar with the arteriole environment, the vWF-mediated mechanism dominates and plays a full role. Large amounts of vWF unfold into long chains under a high fluid shear and adhere to the wound channel, activating and binding a large number of platelets to form a platelet embolism sufficient to block the wound. By contrast, the bond between vWF and platelets is a transient weak bond. First, transient bonds form between inactivated platelets in the blood flow and vWF adheres to the wound surface. The vWF-GP $\mathrm{Ib}$ bonds form rapidly, but also dissociate rapidly, and slow the platelets down, allowing them to roll slowly over the surface of the thrombus [26], allowing time to activate them and enable the formation of longer-lived and strong $\alpha_{\mathrm{IIb}} \beta_{3}$-mediated bonds. The $k_{\text {off }}$ of the vWF-GP bond is usually greater than $3 \mathrm{~s}^{-1}$ [36] and is much larger than that of the integrin-fibrinogen bond. Under high shear conditions, the high dissociation rate of the $\mathrm{vWF}-\mathrm{GP}_{\mathrm{Ib}}$ bond indicates that more 
platelets per unit of time have opportunity to be captured by vWF, slow down to stop on the surface of the wound or form a thrombus, and finally form adhesion an stable and aggregation $[12,36]$. This is the main reason why vWF-mediated mechanisms play a dominant role in high shear.

Although surprisingly, platelet aggregation has difficulty in occurring, and the hemostatic time is prolonged within the intermediate shear range, similar findings have been reported in published studies on intravascular thrombosis. Neeves compared platelet aggregation on Type I collagen at shear rates of 126, 492, and 1,260 s-1 [37] and at 150, 300, 750, and $1,500 \mathrm{~s}^{-1}$ [38] using in vitro models of intravascular thrombosis, and found the platelet aggregation to decrease over 1,000 s' $\mathrm{s}^{-1}$. In addition, Neeves et al. [37, 38] pointed out that this could be the result of competitive effects, i.e., an increase in blood flow increased the platelet flux, but also increased the adhesion bond force load, reduced the interaction time, and diluted the active coagulant substances, resulting in less efficient platelet adhesion and aggregation at higher flow rates. However, these studies did not consider the differences in platelet aggregation mechanisms at different shear rates, nor did they examine the situation in which the shear rate continued to increase. There are some differences between hemostasis and intravascular thrombosis in the blood flow pattern and the thrombus growth environment, although the coagulation mechanism of the two is the same, and thus a similar shearmediated platelet aggregation may occur.

However, in actual physiological situations, the evolution of biological systems does not result in a range of blood flow shear rates that are prone to hemostatic failure. This is because the physiological endovascular environment is much more complex than in the hemostatic model focused solely on platelet aggregation. First, this model only considers the intrinsic coagulation pathway induced by the presence of Type I collagen at the wound site, which is also the most important pathway for platelet adhesion and aggregation [10-12]; however, there are many other pro-coagulant substances in the subendothelial matrix exposed at the vascular wound. Tissue factor (TF) is the most important one. The TF is the initiator of the extrinsic pathway of coagulation, when the
TF is exposed to plasma proteins from the wound, it will bond the clotting factor VII (factor VII, FVII) to trigger a blood coagulation cascade [39, 40.] Therefore, the presence of TF during the actual hemostasis process will also accelerate and enhance the growth of the hemostatic thrombus, thus reducing the hemostasis time. Second, in addition to the above mechanisms, other adhesion receptors and ligands are also involved in platelet aggregation, such as fibronectin. Initially, fibronectin was thought to play a minor role in platelet aggregation, but in vivo studies from $\mathrm{Ni}$ et al. [41] showed that persistent thrombosis was seen in the arterioles of mice lacking vWF and fibrinogen, suggesting a third adhesive ligand involved in platelet aggregation. Subsequent studies in mice with low fibronectin expression have confirmed the important role of fibronectin in promoting platelet aggregation and thrombus growth [30, 42]. Fibrinolytic proteins may cooperatively enhance platelet adhesion, aggregation, and thrombus growth by cross-linking with polymerized fibrin, but their role in shear-dependent platelet aggregation is not fully understood, and therefore its role is not considered in the present study. Finally, in addition to platelet aggregation, the flow shear rate also influences the initial adhesion of platelets at the wound site and subsequent procoagulant action $[26,29,30]$; however, this study only considers the effect of shear-mediated platelet aggregation on the hemostatic process. Therefore, hemostatic failure does not easily occur under normal physiological conditions, whereas the mechanism of shear-mediated platelet aggregation can affect the hemostatic process and hemostatic time to a certain extent, and is likely to have a significant impact under some special pathological conditions (such as vascular hemophilia and other coagulation disorders).

\subsection{Hemostasis under altered microenvironments}

To verify the effect of integrin $\alpha_{\mathrm{II}} \beta_{3}$-fibrinogen and vWF mechanisms on hemostasis under low shear and high shear conditions, the corresponding inhibitors were used to change the blood microenvironments. Tryptopeptide $\left(50 \mu \mathrm{g} \cdot \mathrm{mL}^{-1}\right)$ was added to the whole blood to investigate the effect of integrin $\alpha_{\mathrm{IIb}} \beta_{3}$ on hemostasis at a low shear rate $\left(100 \mathrm{~s}^{-1}\right)$. Eptifibatide is a common anti-platelet agent that inhibits integrin 
$\alpha_{\mathrm{IIb}} \beta_{3}$. As shown in Figs. 4(a1) and 4(a2), in the control group, as soon as platelets adhere and aggregate at the wound site, fibrin rapidly accumulates and grows, eventually forming a stable hemostatic thrombus with a large amount of fibrin and small amounts of platelets, similar with the composition of venous thrombosis [39], and hemostasis was achieved in $15 \mathrm{~min}$. In the eptifibatide-treated group, platelets can still adhere to the collagenous coating of the wound channel as plaques but cannot form clumps of platelet aggregation embolism. Because the platelet surface is the main site of coagulation and fibrin network formation $[2,10]$, the coverage area of fibrin is significantly reduced and is unable to form a sufficiently stable hemostatic thrombus, resulting in hemostatic failure. The results confirm that integrin $\alpha_{\mathrm{II}} \beta_{3}$-mediated platelet aggregation regulates the hemostasis process at low shear rates.

In this study, $30 \mathrm{mM} \mathrm{N}$-Acetyl-L-Cysteine (NAC) was added to the whole blood to investigate the effect of vWF on the hemostasis at a high shear rate $\left(2,000 \mathrm{~s}^{-1}\right)$. NAC is an antioxidant that inhibits

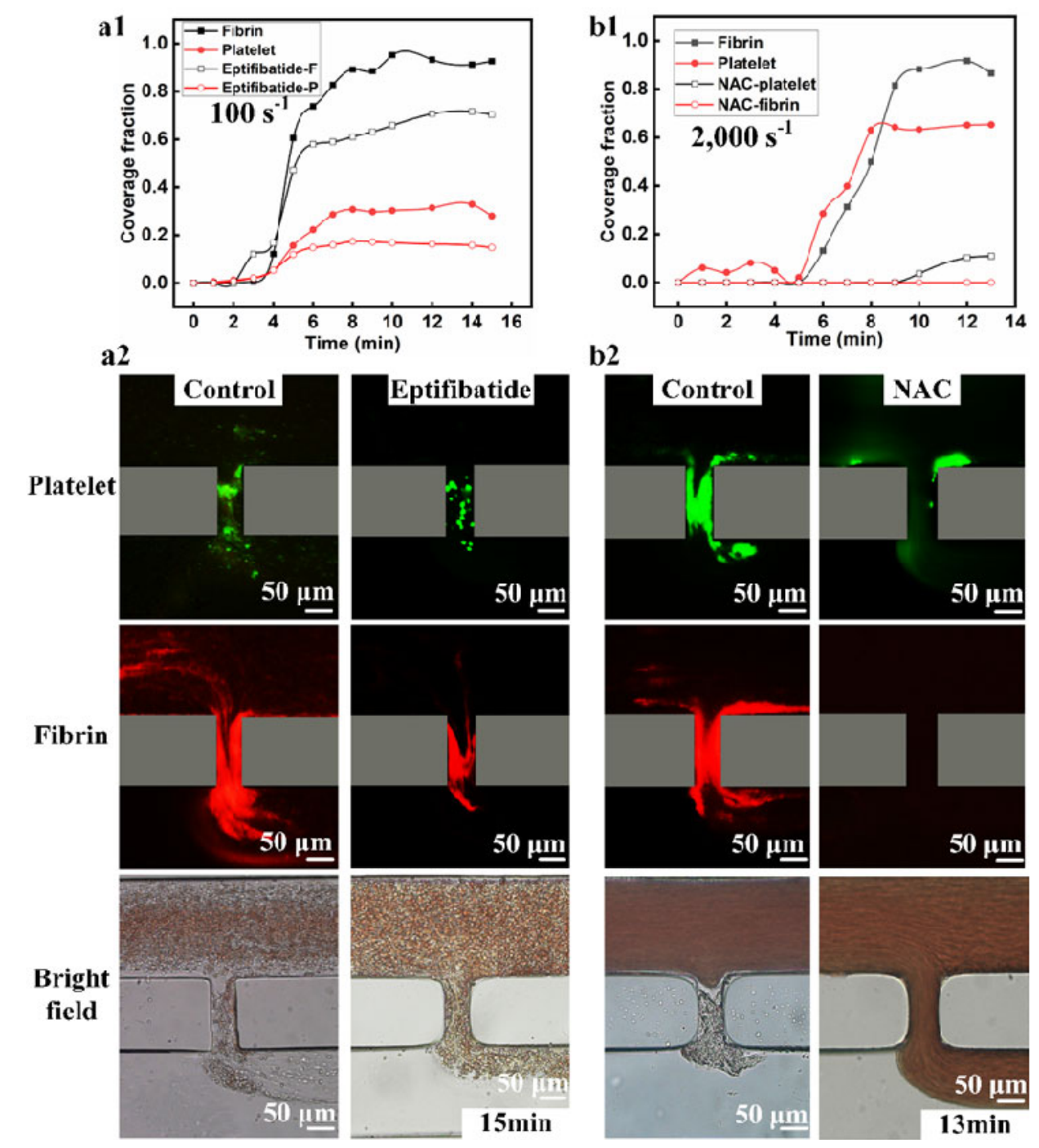

Fig. 4 (a1) At $100 \mathrm{~s}^{-1}$, growth of hemostatic thrombus in the control group and eptifibatide-treated group; (a2) after eptifibatide treatment, platelet aggregation was significantly decreased compared with the control group, indicating that integrin $\alpha_{\text {IIb }} \beta_{3}$ mediates platelet aggregation under low shear conditions and thus affects the hemostasis process; (b1) at 2,000 s ${ }^{-1}$, growth of hemostatic thrombus in the control and NAC-treated groups; (b2) after NAC treatment, platelet aggregation mostly disappeared compared with the control group, indicating that vWF mediates platelet aggregation under high shear conditions and thus affects the hemostasis process. Fluorescently label: platelets (green) and fibrin (ogen) (red), respectively. 
vWF activity by interfering with disulfide bonding, thereby preventing vWF-mediated thrombosis [31]. As shown in Figs. 4(b1) and (b2), in the control group, platelet adhesion and aggregation occurred rapidly in the wound channel after the beginning of the experiment. However, owing to the high blood flow rate and the low local concentration of fibrinogen, the formation of the fibrin network is slow, and the formed platelet embolism is not sufficiently stable and is easy to wash away. Therefore, the platelet coverage area fluctuates within the first $5 \mathrm{~min}$. After $5 \mathrm{~min}$, with the formation of the fibrin network, stable hemostatic thrombus begins to form, and the coverage area of the platelets and fibrin increases rapidly. Finally, hemostatic thrombus rich in platelets and fibrin forms, and hemostasis is achieved within $13 \mathrm{~min}$. In the NAC-treated group, owing to the inhibition of the vWF function, only a small platelet embolism can form at the corner of the passage, and there is almost no platelet distribution inside the wound channel. In the absence of platelets, a fibrin network is difficult to form at such a high shear rate, and the coverage is almost zero, resulting in hemostatic failure. The results further confirm that $\mathrm{vWF}$-mediated platelet aggregation regulates the hemostasis process at high shear rates.

\subsection{Discussion on accelerating hemostasis methods}

It can be seen from the above results that in the intermediate shear rate range $\left(\sim 1,000 \mathrm{~s}^{-1}\right)$, the hemostatic effect of integrin-fibrinogen and a vWF-mediated platelet aggregation mechanism cannot be fully exerted, and thus hemostatic failure is likely to occur. To improve this failure, two approaches can be used to improve the efficiency of hemostasis by adjusting the shear condition of the blood flow at the vascular wound and provide an exogenous coagulant. Conventional methods such as sutures [43], hemostats, sealants, and tissue adhesives [44], can apply pressure to the wound to slow the flow of blood loss and reduce the blood flow shear, thus promoting hemostasis. These methods are suitable for hemostasis of trauma with large wounds, but are not suitable for the microhemorrhage mentioned in this study. Hemostatic gauze and dressings are used for the treatment of a microhemorrhage on the tissue surfaces, which can not only reduce the flow rate of blood loss by applying pressure, but also provide a leakage channel for lost blood owing to its porous nature, further reducing the flow rate of blood loss by dispersing the flow and increasing the contact area of the blood, and thus further promoting hemostasis $[45,46]$. However, the hemostatic devices mentioned above lack the biological activity to activate platelets and trigger a coagulation cascade. Inorganic mesostructured materials, including mesoporous $\mathrm{SiO}_{2}$ [47], mesoporous bioactive glasses [48], microporous aluminosilicates [49], and their composites have recently been widely used in the field of hemostasis owing to their highly mesoporous structure and procoagulation surface. Biomaterials with biocoagulation-promoting activities, such as gelatin [50,51] and chitosan [52, 53], have been developed into various forms of hemostatic materials such as sponges, hydrogels, and nanoparticles, and have been widely used depending on their rapid activation of the platelets and coagulation cascade reactions [50-53]. The cold atmospheric plasma (CAP), which contains an abundant reactive oxygen species, active nitrogen, and other active groups that can promote blood coagulation, allows for effective blood coagulation without thermal effects, and has wide application potential in the treatment of a microhemorrhage such as an errhysis and a gastrointestinal hemorrhage [54, 55].

\section{Conclusions}

In this study, a microfluidic bleeding model was developed to investigate the effect of blood flow shear on microvascular hemostasis. The blood flow is controlled to mimic the flow shear bioenvironment from the venous to the arteriole. The presence of the intermediate shear rate, which is prone to hemostasis failure, suggests that microvascular hemostasis is influenced and regulated by the flow shear. Based on the growth process and composition distribution of hemostatic thrombus, it was found that blood shear can affect the growth and morphology of hemostatic thrombus by regulating the platelet aggregation, and finally regulate the hemostatic 
process. At low shear rates, the dominant mechanism of platelet aggregation is the integrin $\alpha_{\mathrm{IIb}} \beta_{3}$-fibrinogen interaction. At a high shear rate, the vWF-mediated mechanism dominates. At an intermediate shear rate, the scouring of faster blood flow makes it difficult for fibrinogen and other coagulants to maintain a relatively high concentration, and the integrin $\alpha_{\mathrm{IIb}} \beta_{3}$-fibrinogen mechanism has difficulty in playing a good role. This shear rate also makes it difficult to apply the vWF-mediated mechanism. As a result, it is difficult for platelets to aggregate into embolisms that can block the entire channel, and hemostasis failure is likely to occur. The results of the hemostasis process with an altered microenvironment using two inhibitors, eptifibatide and NAC, further confirmed the shear-mediated dominant platelet aggregation mechanism. Consequently, the microfluidic bleeding model in this study may be used to study the hemostasis mechanism in different physiological environments, which is of significance for the reliable prediction of a microhemorrhage and an adjustment of the treatment scheme.

\section{Acknowledgements}

This study was supported by the National Natural Science Foundation of China (No. 51875304) and the National Key R\&D Program of China (No. 2018YFE0114900).

Open Access This article is licensed under a Creative Commons Attribution 4.0 International License, which permits use, sharing, adaptation, distribution and reproduction in any medium or format, as long as you give appropriate credit to the original author(s) and the source, provide a link to the Creative Commons licence, and indicate if changes were made.

The images or other third party material in this article are included in the article's Creative Commons licence, unless indicated otherwise in a credit line to the material. If material is not included in the article's Creative Commons licence and your intended use is not permitted by statutory regulation or exceeds the permitted use, you will need to obtain permission directly from the copyright holder.
To view a copy of this licence, visit http://creativecommons.org/licenses/by/4.0/.

\section{References}

[1] Hoffman M, Monroe D M. Coagulation 2006: A modern view of hemostasis. Oncol Clin $N$ Am 21(1): 1-11 (2007)

[2] Colace T V, Tormoen G W, McCarty O J T, Diamond S L. Microfluidics and coagulation biology. Annu Rev Biomed Eng 15: 283-303 (2013)

[3] Gralnek I M, Barkun A N, Bardou M. Management of acute bleeding from a peptic ulcer. N Engl J Med 359(9): 928-937 (2008)

[4] Lisman T, Porte R J. Rebalanced hemostasis in patients with liver disease: Evidence and clinical consequences. Blood 116(6): 878-885 (2010)

[5] Kim B J, Lee S H. Cerebral microbleeds: Their associated factors, radiologic findings, and clinical implications. J Stroke 15(3): 153 (2013)

[6] Qureshi A I, Tuhrim S, Broderick J P, Batjer H H, Hondo H, Hanley D F. Spontaneous intracerebral hemorrhage. N Engl J Med 344(19): 1450-1460 (2001)

[7] Shoeb M, Fang M C. Assessing bleeding risk in patients taking anticoagulants. J Thromb Thrombolysis 35(3): 312-319 (2013)

[8] Altintas O, Niftaliyev E, Asil T. The relationship between brain microbleeds and homeostatic markers in the treatment of ischemic stroke. Neurol Res 40(12): 1048-1053 (2018)

[9] Ziliotto N, Zivadinov R, Jakimovski D, Bergsland N, Ramasamy D, Weinstock-Guttman B, Ramanathan M, Marchetti G, Bernardi F. Are plasma levels of vascular adhesion protein-1 associated both with cerebral microbleeds in multiple sclerosis and intracerebral haemorrhages in stroke? Thromb Haemost 119(1): 175-178 (2019)

[10] Zilberman-Rudenko J, McCarty O J T. Utility and development of microfluidic platforms for platelet research. Platelets 28(5): 425-426 (2017)

[11] Farndale R W, Sixma J J, Barnes M J, de Groot P G. The role of collagen in thrombosis and hemostasis. $J$ Thromb Haemost 2(4): 561-573 (2004)

[12] Fogelson A L, Neeves K B. Fluid mechanics of blood clot formation. Annu Rev Fluid Mech 47(1): 377-403 (2015)

[13] Colace T V, Diamond S L. Direct observation of von willebrand factor elongation and fiber formation on collagen during acute whole blood exposure to pathological flow. Arterioscler Thromb Vasc Biol 33(1): 105-113 (2013) 
[14] Kratzer M A A, Born G V R. Simulation of primary haemostasis in vitro. Pathophysiol Haemos Thromb 15(6): 357-362 (1985)

[15] Branchford B R, Ng C J, Neeves K B, di Paola J. Microfluidic technology as an emerging clinical tool to evaluate thrombosis and hemostasis. Thromb Res 136(1): 13-19 (2015)

[16] Robert W C. Hemostasis and Thrombosis: Basic Principles and Clinical Practice. 5th edn. Philadelphia (USA): Lippincott Williams \& Wilkins, 2006.

[17] Mackman N. Tissue-specific hemostasis in mice. Arterioscler Thromb Vasc Biol 25(11): 2273-2281 (2005)

[18] Yamashita A, Asada Y. A rabbit model of thrombosis on atherosclerotic lesions. J Biomed Biotechnol 2011: 1-15 (2011)

[19] Wong K H K, Chan J M, Kamm R D, Tien J. Microfluidic models of vascular functions. Annu Rev Biomed Eng 14(1): 205-230 (2012)

[20] Harris L F, Killard A J. Microfluidics in coagulation monitoring devices: A mini review. Anal Methods 10(30): 3714-3719 (2018)

[21] Zhang Y S, Oklu R, Albadawi H. Bioengineered in vitro models of thrombosis: Methods and techniques. Cardiovasc Diagn Ther 7(S3): S329-S335 (2017)

[22] Neeves K B, Onasoga A A, Hansen R R, Lilly J J, Venckunaite D, Sumner M B, Irish A T, Brodsky G, Manco-Johnson M J, di Paola J A. Sources of variability in platelet accumulation on type 1 fibrillar collagen in microfluidic flow assays. PLoS One 8(1): e54680 (2013)

[23] Schoeman R M, Rana K, Danes N, Lehmann M, di Paola J A, Fogelson A L, Leiderman K, Neeves K B. A microfluidic model of hemostasis sensitive to platelet function and coagulation. Cel Mol Bioeng 10(1): 3-15 (2017)

[24] Sakurai Y, Hardy E T, Ahn B, Tran R, Fay M E, Ciciliano J C, Mannino R G, Myers D R, Qiu Y Z, Carden $\mathrm{M} \mathrm{A}$, et al. A microengineered vascularized bleeding model that integrates the principal components of hemostasis. Nat Commun 9: 509 (2018)

[25] Gogia S, Neelamegham S. Role of fluid shear stress in regulating VWF structure, function and related blood disorders. Biorheology 52(5-6): 319-335 (2016)

[26] Maxwell M J, Westein E, Dopheide S M, Jackson S P. Identification of a two-stage platelet aggregation process mediating shear-dependent thrombus formation. Vasc Pharmacol 45(3): 189 (2006)

[27] Shankaran H, Alexandridis P, Neelamegham S. Aspects of hydrodynamic shear regulating shear-induced platelet activation and self-association of von Willebrand factor in suspension. Blood 101(7): 2637-2645 (2003)

[28] McDonald J C, Duffy D C, Anderson J R, Chiu D T, Wu H K, Schueller O J A, Whitesides G M. Fabrication of microfluidic systems in poly (dimethylsiloxane). Electrophoresis 21(1): 27-40 (2000)

[29] Sarratt K L, Chen H, Zutter M M, Santoro S A, Hammer D A, Kahn M L. GPVI and $\alpha_{2} \beta_{1}$ play independent critical roles during platelet adhesion and aggregate formation to collagen under flow. Blood 106(4): 1268 1277 (2005)

[30] Jackson S P. The growing complexity of platelet aggregation. Blood 109(12): 5087-5095 (2007)

[31] Wong K H K, Edd J F, Tessier S N, Moyo W D, Mutlu B R, Bookstaver L D, Miller K L, Herrara S, Stott S L, Toner M. Anti-thrombotic strategies for microfluidic blood processing. Lab Chip 18(15): 2146-2155 (2018)

[32] Gralnick H R, Williams S B, Coller B S. Fibrinogen competes with von Willebrand factor for binding to the glycoprotein IIb/IIIa complex when platelets are stimulated with thrombin. Blood 64(4): 797-800 (1984)

[33] Shiba E, Lindon J N, Kushner L, Matsueda G R, Hawiger J, Kloczewiak M, Kudryk B, Salzman E W. Antibody-detectable changes in fibrinogen adsorption affecting platelet activation on polymer surfaces. $\mathrm{Am} J$ Physiol-Cell Physiol 260(5): C965-C974 (1991)

[34] Pries A R, Neuhaus D, Gaehtgens P. Blood viscosity in tube flow: Dependence on diameter and hematocrit. $\mathrm{Am}$ J Physiol-Heart Circ Physiol 263(6): H1770-H1778 (1992)

[35] Litvinov R I, Barsegov V, Schissler A J, Fisher A R, Bennett J S, Weisel J W, Shuman H. Dissociation of bimolecular $\alpha_{\mathrm{IIb}} \beta_{3}$-fibrinogen complex under a constant tensile force. Biophys $J$ 100(1): 165-173 (2011)

[36] Yago T, Lou J Z, Wu T, Yang J, Miner J J, Coburn L, López J A, Cruz M A, Dong J F, McIntire L V, et al. Platelet glycoprotein Ib $\alpha$ forms catch bonds with human WT vWF but not with type 2B von Willebrand disease vWF. J Clin Invest 118(9): 3195-3207 (2008)

[37] Neeves K B, Maloney S F, Fong K P, Schmaier A A, Kahn M L, Brass L F, Diamond S L. Microfluidic focal thrombosis model for measuring murine platelet deposition and stability: PAR4 signaling enhances shear-resistance of platelet aggregates. J Thromb Haemost 6(12): 2193-2201 (2008)

[38] Neeves K B, Onasoga A A, Hansen R R, et al. Sources of variability in platelet accumulation on type 1 fibrillar collagen in microfluidic flow assays. Plos One 8(1): e54680-54690 (2013).

[39] Govindarajan V, Zhu S, Li R Z, Lu Y C, Diamond S L, Reifman J, Mitrophanov A Y. Impact of tissue factor localization on blood clot structure and resistance under venous shear. Biophys J 114(4): 978-991 (2018)

[40] Mann K G, Krudysz-Amblo J, Butenas S. Tissue factor controversies. Thromb Res 129: S5-S7 (2012)

[41] Ni H, Yuen P S T, Papalia J M, Trevithick J E, Sakai T, 
Fassler R, Hynes R O, Wagner D D. Plasma fibronectin promotes thrombus growth and stability in injured arterioles. PNAS 100(5): 2415-2419 (2003)

[42] Matuskova J, Chauhan A K, Cambien B, Astrof S, Dole V S, Piffath C L, Hynes R O, Wagner D D. Decreased plasma fibronectin leads to delayed thrombus growth in injured arterioles. Arterioscler Thromb Vasc Biol 26(6): 1391-1396 (2006)

[43] Duarte A P, Coelho J F, Bordado J C, Cidade M T, Gil M H. Surgical adhesives: Systematic review of the main types and development forecast. Prog Polym Sci 37(8): 1031-1050 (2012)

[44] Achneck H E, Sileshi B, Jamiolkowski R M. A comprehensive review of topical hemostatic agents: Efficacy and recommendations for use. Ann Surg 251(2): 217-228 (2010)

[45] Liang D H, Lu Z, Yang H, Gao J T, Chen R. Novel asymmetric wettable AgNPs/chitosan wound dressing: In vitro and in vivo evaluation. ACS Appl Mater Interfaces 8(6): 3958-3968 (2016)

[46] Biranje S S, Madiwale P V, Patankar K C, Chhabra R, Dandekar-Jain P, Adivarekar R V. Hemostasis and antinecrotic activity of wound-healing dressing containing chitosan nanoparticles. Int $J$ Biol Macromol 121: 936-946 (2019)

[47] Pourshahrestani S, Kadri N A, Zeimaran E, Towler M R. Well-ordered mesoporous silica and bioactive glasses: Promise for improved hemostasis. Biomater Sci 7(1): 31-50 (2019)

[48] Baino F, Fiume E. 3D printing of hierarchical scaffolds based on mesoporous bioactive glasses (MBGs)Fundamentals and applications. Materials 13(7): 1688 (2020)



Yongjian LI. He received his Ph.D. degree in mechanical engineering from Tsinghua University, China, in 2009. He joined the Department of Mechanical Engineering at

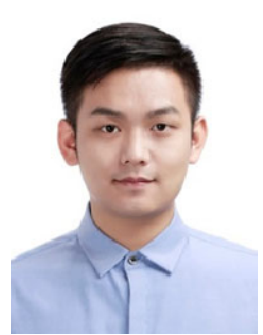

[49] Pourshahrestani S, Zeimaran E, Djordjevic I, Kadri N A, Towler M R. Inorganic hemostats: The state-of-the-art and recent advances. Mater Sci Eng: C 58: 1255-1268 (2016)

[50] Zhao X, Guo B L, Wu H, Liang Y P, Ma P X. Injectable antibacterial conductive nanocomposite cryogels with rapid shape recovery for noncompressible hemorrhage and wound healing. Nat Commun 9: 2784 (2018)

[51] Zhang Z Y, Kuang G Z, Zong S, Liu S, Xiao H H, Chen X S, Zhou D F, Huang Y B. Sandwich-like fibers/sponge composite combining chemotherapy and hemostasis for efficient postoperative prevention of tumor recurrence and metastasis. Adv Mater 30(49): 1803217 (2018)

[52] Zhou Y P, Li H Y, Liu J W, Xu Y, Wang Y L, Ren H, Li $\mathrm{X}$ M. Acetate chitosan with $\mathrm{CaCO}_{3}$ doping form tough hydrogel for hemostasis and wound healing. Polym $A d v$ Technol 30(1): 143-152 (2019)

[53] Biranje S S, Madiwale P V, Patankar K C, Chhabra R, Dandekar-Jain P, Adivarekar R V. Hemostasis and anti-necrotic activity of wound-healing dressing containing chitosan nanoparticles. Int J Biol Macromol 121: 936-946 (2019)

[54] Musavi E S, Khorashadizadeh S M, Fallah R, Rahmanian Sharifabad A. Effect of nonthermal atmospheric pressure plasma on plasma coagulation in healthy persons and patients under treatment with Warfarin. Contributions Plasma Phys 59(3): 354-357 (2019)

[55] Kurosawa M, Takamatsu T, Kawano H, Hayashi Y, Miyahara H, Ota S, Okino A, Yoshida M. Endoscopic hemostasis in porcine gastrointestinal tract using $\mathrm{CO}_{2}$ low-temperature plasma jet. J Surg Res 234: 334-342 (2019)

Tsinghua University in 2011. His current position is as an assistant professor. His research areas cover the tribology in seals and bearings, transport phenomena, and interfacial behaviors in microfluidics and bio-chips.

a Ph.D. candidate in mechanical engineering in Tsinghua University, China. His research areas are microfluidics and bio-chips. 